\title{
Comparative Study between Intra-medullary Nailing and Minimally Invasive Plate Osteosynthesis (MIPO) in Closed Distal Tibia Fracture in a District Hospital in Bangladesh
}

\author{
Rahman MA ${ }^{1}$, Kaiser $\mathrm{MS}^{2}$, Rokonuzzaman $\mathrm{SM}^{3}$, Ahmed $\mathrm{N}^{4}$
}

Conflict of Interest: None

Received: 02.03 .2020

Accepted: $05.05-.2020$

www.banglajol.info/index.php/JSSMC

Key Words: Intra-medullary Nailing, Minimally Invasive Plate Osteosynthesis (MIPO), Closed Distal Tibia Fracture

\begin{abstract}
Introduction: Tibia fractures are the most common long bone fractures encountered by the orthopedic surgeons and distal tibia fractures have the second highest incidence of all tibia fractures after the middle third of tibia the distal tibial fractures are unique and are considered as most challenging fractures to treat due to its proximity to the ankle joint and its superficial nature. The objective of this study is to compare two osteosynthesis systems developed for surgical treatment of distal tibia fractures: the intramedullary nailing and the MIPO technique.
\end{abstract}

Methods: The study was conducted between Jan 2018 to Dec 2019. 30 patients with extraarticular distal tibia fracture treated with intramedullary nailing and MIPO technique were reviewed retrospectively and clinical outcome was evaluated according to American Orthopaedic Foot and Ankle Score.

Results: 15 patients were treated with intramedullary nail and 15 with MIPO technique. Fibular fixation was done in cases where fibular fracture was at or below the level of tibial fractures. We found no difference in terms of time for fracture union, mal-union, non-union, duration of surgery and amount of blood loss. But there was significant difference in terms of infection and duration of hospital stay. Also weight bearing was possible much earlier in intramedullary group as compared to the MIPO group.

Conclusion: Thus we conclude that intramedullary nailing is better choice of implant in patients with extra- articular distal tibia fractures and helps in early weight bearing and ambulation of patient with fewer complications.

[J Shaheed Suhrawardy Med Coll 2020; 12(1): 33-37] DOI: https://doi.org/10.3329/jssmc.v12i1.51616

\section{Introduction}

Fractures of distal tibia metaphysis occur typically as a result of axial and rotational forces on lower extremity and represents approximately $10 \%$ of the fractures of distal end of tibia. ${ }^{1,2}$ The degree of associated soft tissue injury is higher in distal metaphyseal fractures than with shaft fractures. ${ }^{3}$ This fracture can be treated by plating or with closed reduction and reamed intramedullary nailing. However, two of the most used techniques are locked intra-

1. Dr. Md. Asjadur Rahman, Asst. Professor, Department of Orthopaedics, Sheikh Hasina Medical College, Tangail

2. Dr. Md. Shahidullah Kaiser, Assoc. Professor and Head, Department of Orthopaedics, Sheikh Hasina Medical College, Tangail

3. Dr. S.M. Roknuzzaman, Assistant Professor, Department of Orthopaedics, Sheikh Hasina Medical College, Tangail

4. Dr. Nadim Ahmed, Senior Consultant, Department of Surgery, Shaheed Suhrawardy Medical College, Dhaka

Correspondence to: Dr. Md. Asjadur Rahman, Asst. Professor, Department of Orthopaedics, Sheikh Hasina Medical College, Tangail, Mobile: +8801715312987, E-mail: dr.a.rahman73@gmail.com medullary interlocking nail and minimally invasive plate osteosynthesis (MIPO). Open plating of the medial aspect of distal tibia caused a statistically significant greater disruption of extraosseous blood supply of the metaphyseal area than percutaneous applied plates. ${ }^{4}$ The best treatment for displaced, extra-articular fractures of the distal tibia (specifically the lower third of the tibia) remains controversial. These injuries are particularly difficult to manage due to the limited soft tissue coverage, poor vascularity of the area, and proximity of the fracture to the ankle joint. Infections, nonunion, and malunion are well-recognized complications of fractures of the distal tibia also, there is difûculty in maintenance of reduction, and thus management of these fractures is very controversial; in the more distal metaphyseal region of the tibia, the nail fixation may be less stable. ${ }^{5}$ The bolts or screws that are inserted into the nail may break ${ }^{6}$, malalignment may occur ${ }^{7}$, and there is a risk that the nail 
will penetrate into the ankle The Fixation of Distal Tibia Fractures (FixDT)trial was designed to compare intramedullary nail fixation with locking plate fixation for adult patients with a displaced fracture of the distal tibial joint ${ }^{8,9}$, Intra-medullary nails for distal tibia fractures are technically difûcult to perform and must be addressed with care. Also, the maintenance of reduction of proximal and distal metaphysical fractures can be difûcult to control with an intramedullary device, leading to mal-alignment; rates varying from $5 \%$ to $58 \%$. Failures in controlling distal fragments may lead to deformities and problems in union like mal-union and non-union. Intra-medullary nailing of the tibia has also been associated with knee pain in up to $71 \%$ of patients. ${ }^{4}$ But, the advantages of closed intramedullary nailing are that it minimizes surgical insult to the fracture and adjacent soft tissues have been associated with shorter time to union and a shorter period of disability. MIPO is an alternative treatment for these fractures, because it does not need large exposure for reduction, thus providing biological ûxation. However, failure to achieve satisfactory reduction may result in angular deviations, non-union and pseudoarthrosis. ${ }^{9}$

\section{Objectives}

The objective of the study was to set the indications for both modalities of fixation in various fracture subtypes; to compare the functional outcomes of distal metaphyseal fractures of tibia managed by both methods at 2 years; and to study complication rates with different modalities of fixation.

\section{Materials and methods}

This is prospective study conducted in Department of Orthopaedics, 250 Bedded General Hospital, Tangail between January 2018 and December 2019. The study consisted of 30 patients' metaphyseal fracture of tibia with either reamed intramedullary nailing or locked plating with open reduction method or minimally invasive techniques. Biplanar injury radiography was evaluated to determine the fracture location and involvement of distal part of tibia.

\section{Inclusion Criteria:}

Skeletally mature patient, involving distal $5 \mathrm{~cm}$ of tibia (AO types A1, A2, A3 and B1) and all closed and GustiloAnderson type 1 fractures.

\section{Exclusion Criteria:}

Skeletally immature patient and Fractures with intraarticular extension (AO type B, C2, C3) 3), Open fractures of Gustilo-Anderson type 2\&3, all cases with DM where excluded. The eligible patients were randomly divided into two group, Group 1 includes patients managed with closed reduction and reamed nailing and Group 2 includes patients treated with locked plating techniques by minimally invasive method (MIPO). The patients in both groups were matched for various confounding variables (age, sex, fracture types, comorbid condition, and associated fractures) and fifteen patients in each group were selected for final outcome study. Follow ups took place at 6 weeks and 3, 5, 7,9 and 12 months after surgery with clinical and radiological examination A distal tibial fracture was defined as a fracture extending within 2"Müllersquares" of the ankle joint. The criterion for inclusion was the presence of a distal fragment of at least $2.5 \mathrm{~cm}$ in length, with no articular incongruity and located within $10 \mathrm{~cm}$ from the tibial plafond. 7Patients were excluded if the fracture was open, pathological fractures, the fracture extended into the ankle joint, there was a contraindication to intramedullary nail fixation (eg, a total knee replacement or previous deformity of the tibia), there was a contraindication to anesthesia, or the patient was unable to complete questionnaires. All patients gave written informed consent. Tscherne classiûcation was used to assess the extent of the soft tissue injuries in closed fractures.

For intramedullary nail fixation, an intramedullary nail is inserted at the proximal end of the tibia and passed down the hollow center (medullary canal) of the bone to hold the fracture in the correct anatomical position. The reduction technique, the surgical approach, the type and size of the nail, the configuration of the proximal and distal interlocking screws, and any supplementary device or technique was left to the discretion of the surgeon as per standard clinical practice. For locking plate fixation, a locking plate is inserted at the distal end of the tibia and passed under the skin onto the surface of the bone. The associated ûbular fracture was ûxed either with plate or intra-medullary nail, only if it was at or below the level of tibial fractures. Fixed-angle screws must be used in at least some of the distal screw holes - this is standard practice with all distal tibia locking plates.

Non weight bearing was recommended for at least 8 weeks post-operatively for both groups. Surgeon discretion was used to determine progression of weight bearing based on fracture pattern, soft tissue injury, bone quality and patient comorbidities. Patients were followed up for a minimum period of 12 months post-operatively. Patients were evaluated with radiographs in both anteroposterior and lateral views of ankle joint and distal tibia preoperatively, immediate post-operatively and at approximately 6 week intervals thereafter until fracture union. Mal-union was deûned as 5 degree of angular deformity or translation/ shortening of $10 \mathrm{~mm}$. Fracture union was deûned clinically as no pain or tenderness at the fracture site and radiologically as healing of at least three of four cortices on bi-planar plain radiographs. Delayed union was deûned as lack of any healing on plain radiographs within 3 
months. Non-union was deûned as lack of any healing on plain radiographs within 6 months. Local complications included infection, vascular and neurological injury, venous thromboembolism, and malunion. At the end of follow-up (after 1 year), clinical outcome was evaluated according to the American Orthopedic Foot and Ankle score as excellent (90e100), good (75e89), fair (50e74) and poor $(<50)$.

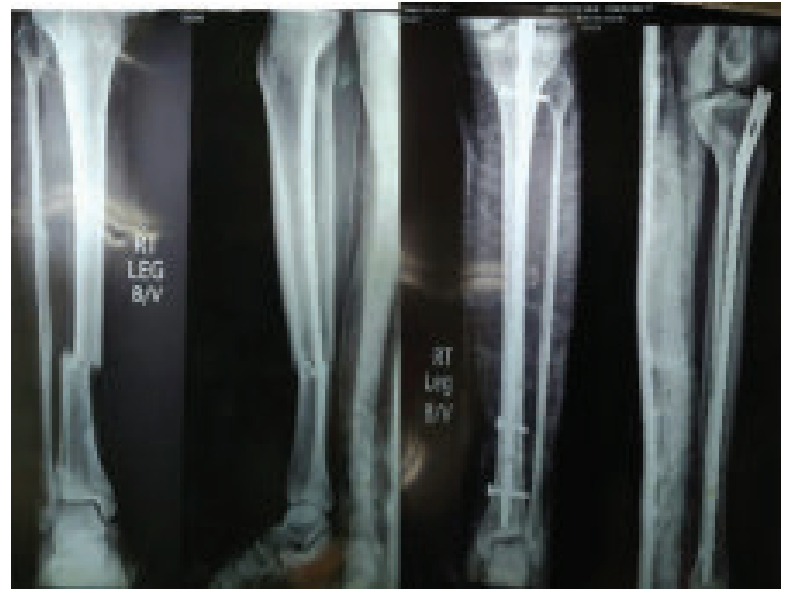

Fig. 1 : ORIF by interlocking nail

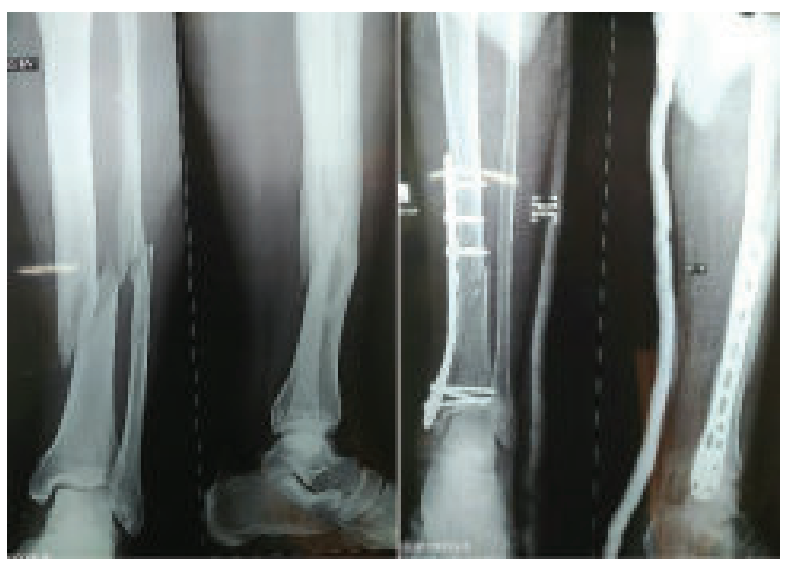

Fig. 2 : MIPO by distal tibial locking plate

\section{Statistical analysis}

The two groups were compared with respect to age, sex, Arbeitsgemeinschaft für Osteosynthesefragen (AO) fracture type, number of closed and open fractures (Gustilo-Anderson type I), pre-operating waiting period, operating time, duration of hospital stay, bony union time, incidence of wound complications, malunion and nonunion of the fracture and the American Orthopedic Foot and Ankle score. Unpaired Student $t$ test and uncorrected c2 test were used as the statistical test, and analysis was performed using "open EPI" online site (data analysis online site). The chosen level of signiûcance was $\mathrm{p}<0.05$.

\section{Results}

Partial weight-bearing ambulation was started at 8 weeks in the interlocking intra-medullary nail group as compared with12weeks in the MIPO group $(p=0.01)$. Fractures of tibial distal third are difûcult to treat. When associated with a poor lining of soft tissues, these fractures are frequently comminuted or present a small metaphyseal fragment. Traditional techniques fail to achieve an appropriate reduction and maintenance of fractures or may further damage soft parts. Biological ûxation of tibial distal third fractures is beneûcial and technically feasible. The advantages are as follows: it reduces injuries on soft parts, it does not compromise bone vascularization and presents a low complication rate, especially when compared with open reduction and internal ûxation. These fractures, when not involving joints, may be treated by two different manners: locked intra-medullary nail or bridge plate using a minimally invasive technique.5 In this study, we found that the cost of treatment for the intramedullary nail group was lower as compared with the MIPO group. Also, the longer duration of hospital- stay in the MIPO group further increased the cost of treatment.

In randomized prospective study of 85 distal tibia fractures by Guoetal, 9 the operation was delayed for upto10days if the leg was considerably swollen and bruised when plating was used as the treatment modality in contrast intra-medullary nailing was associated with earlier operation. In the present study, the preoperative waiting period was 6 days as compared with 3 days for the interlocking nail group which was found statistically signiûcant. Their study also concluded that the mean radiation time and operating time were signiûcantly longer in the minimally invasive locked compression plate group as compared with intra-medullary nail group, which was also the case in the present study. Thus, there was longer duration of hospital stay in the MIPO group as compared with the intra-medullary nailing group adding to the cost of treatment. Lietal10 in their retrospective study including 46 patients found that the mean operating time and mean duration of hospital stay was $76.1 \pm 16.6$ minutes and 5.8 \pm 2.1 days, respectively, for the interlocking nail group was signiûcantly shorter when compared with $90.4 \pm 20.3$ minutes and $8.9 \pm 3.1$ days, respectively, in the MIPO group. Also, the time for full weight bearing was $11.1 \pm$ 1.7 weeks in the MIPO group, which was signiûcantly longer than $9.0 \pm 1.4$ weeks in the interlocking nail group. So, they concluded that multidirectional locked nailing may be considered a better surgical option as it offers advantages in terms of mean operating time, hospital stay and full weight-bearing time. 
Surgical treatment for distal tibial metaphyseal fractures is a challenge because extensive soft-tissue injuries often disrupt the vascular supply to the fracture site and increase the risk of infection and delayed union or non-union because of the thin soft tissue envelop and precarious blood supply in this region. The American Orthopaedic Foot and Ankle surgery scoring system was used by Guo et a [10] and the Olerud-Molander Ankle Score by Li et al [11] to evaluate the functional results, respectively; both the studies showed no statistically signiûcant difference between the functional outcome of either intra-medullary interlocking or MIPO group. Thus, suggesting that closed intramedullary interlocking nailing and a minimally invasive percutaneous plate osteosynthesis can be used safely and effectively to treat distal metaphyseal fractures of the tibia.

\section{Discussion}

Both groups were comparable with regards to age, sex and fracture types. Mean age of cases was 40 years in group 1 compared to 42 years in group 2. Most common mode of trauma in both groups was road traffic accident (50\% cases) followed by sports related injury in group $1(20 \%$ cases ) and fall from height in group 2 (15\% cases). This multicenter trial of patients with a fracture of the distal tibia found no significant difference between intramedullary nail fixation and locking plate fixation in disability rating at 6 months or 12 months.

The average duration of surgery in group 2 was 94 minutes (range,70-120 minutes) whereas average duration of surgery in group 1 was 85 minutes (range,65 to 105 minutes $),(p=0.21)$ meaning that difference between 2 groups regarding duration of surgery is not significant. No significant intraoperative complications were noted in both groups. Difficult reduction was observed in 3 patients in group 1 and 4 patients in group 2. Proximity to ankle joint amplifies the bending moment of the distal segment and may facilitate fracture propagation into the ankle joint. No such complication was observed in our cases.

Average time after which patient was allowed full weight bear on the operated limb was 14.25 weeks (range,11-18 weeks) in group1 and 17.32 weeks (range,14-22 weeks) in group $2,(p=0.001)$. The cases in group 1 were able to bear weight on the operated limb in significant earlier time and able to perform independent activities at earlier time compared to group 2.

There was no significant difference in union time between two groups. Higher rate of delayed union was found in group 2(20\%) compared to group 1(10\%). Two patients in group 1 failed to achieve union by 24 weeks. One of them required autogenous bone grafting and went to union by 34 weeks and other patient required dynamization with bone grafting and went to union by 36 weeks.

Angular deformity was found in $15 \%$ of patients in group 1 and $10 \%$ of patients in group2. Mean angulation in group 1 was 3.4 degree (range, 0-12) and 1.0 degree (range,0-9) in group $2(p=0.04)$. Varus deformity of $>5$ degree was found in 4 cases and valgus deformity $>5$ degree in single case in group 1. 2 cases in group 2 had deformity $>5$ degree, one had varus and other had valgus deformity. This shows that angular malalignments were more common in group1. This complication may initially result from an incorrect entry site and entrance angle. In addition, the incidence of malalignment may be caused by instability due to the large difference between the diameter of the implant and medullary cavity of distal metaphysis. The nail does not contact the metaphyseal cortex; therefore it may translate along the interlocking screws unless anteroposterior locking is also performed. The use of Pollar screws has been described as additional stabilization technique and reduction tool to increase the strength of bone implant construct.

Malalignment can lead to complaints from the patient with regard to walking, practicing sports and so forth. Puno et al evaluated 27 patients with 28 tibial fractures at an average of 8.2 years (range, 612.5 years). They found a correlation joint malalignment and clinical outcome for fractures of tibia. Analysis showed that a greater degree of ankle malalignment produces poor clinical outcomes. ${ }^{12}$ Long term effect of malalignment needs to be evaluated further.

Shortening $(>1 \mathrm{~cm})$ was found in $20 \%$ of patients in group 1 and $10 \%$ of patients in group 2 . Average shortening was $0.8 \mathrm{~cm}$ (range, $0-1.5$ ) in group1 and $0.3 \mathrm{~cm}$ (range, $0-1.3$ ) in group $2(\mathrm{p}=0.012)$. clinically significant shortening was found in only 2 cases treated with intramedullary nailing and one case treated with plating.

Most important postoperative complication in nailing group anterior knee pain and postoperative infection patients treated with plating group. Incidence of anterior knee pain in nailing cases was $30 \%$. This complication is seen exclusively in nailing group. Operative site infection was seen in 5\% (all superficial) cases in group 1 and 15\% (all superficial) cases in group 2. Superficial infection subsided with intravenous antibiotics and continued dressing, and healed eventually.

Patients treated with intramedullary nailing have statistically better ankle range of motion. Average ankle dorsiflexion was 15 degree and plantarflexion 22 degree in nailing group compared to dorsiflexion of 9 degree and plantarflexion of 13 degree in plating group. 
Higher average functional ankle score was found in nailing group compared to plating group. The average Olerud and Molander score was 86 (range,75-93) in group 1 and 80 (range, 59-93) in group $2(\mathrm{p}=0.008)$.

\section{Conclusion}

Thus, we conclude that interlocking intra-medullary nailing and MIPO are effective options in the management of distal tibia fractures, but interlocking nailing fares better than MIPO with regards to early weight bearing, less preoperative waiting period, shorter ambulation and wound complications. Also, as the implant is cheaper, there is less preoperative waiting and shorter duration of hospital stay. Closed intra-medullary nailing is a potentially costeffective alternative to MIPO for the treatment of distal tibia fractures. Among patients16yearsorolderwithanacute, displaced, extra-articular fracture of the distal tibia, neither nail fixation nor locking plate fixation resulted in superior disability status at 6 months. Other factors may need to be considered in deciding the optimal approach.

Due to small number of patients involved in our study, we cannot draw any definitive conclusions from our preliminary results but view them as valuable basis for further studies. Further research is necessary, in order to evaluate whether this surgical technique in long term provides us with the safe and effective management options for distal tibia fractures.

\section{References}

1. Court-Brown CM, McBirnie J. The epidemiology of tibial fractures. J Bone Joint Surg Br. 1995 May;77(3):417e21.

2. Blick SS, Brumback RJ, Lakatos R, Poka A, Burgess AR. Early prophylactic bone grafting of high-energy tibial fractures. Clin Orthop Relat Res 1989 Mar;(240): 21e41.
3. Iqbal HJ, Pidikiti P. Treatment of distal tibia metaphyseal fractures; plating versus intramedullary nailing: a systematic review of recent evidence. Foot Ankle Surg 2013 Sep;19(3):143e7.

4. Vallier HA, Le TT, Bedi A. Radiographic and clinical comparisons of distal tibia shaft fractures (4 to $11 \mathrm{~cm}$ proximal to the plafond): plating versus intramedullary nailing. J Orthop Trauma 2008 MayJun; 22(5):307e11.

5. Fan CY, Chiang CC, Chuang TY, et al. interlocking nails for displaced metaphyseal fractures of distal tibia. Injury, 2005; 36; 669-674.

6. Ovadia DN, Beals RK. The fractures of tibial plafond. J of bone joint surgery America. 1986;68; 543-551.

7. Robinson CM, McLauchlan GJ, McLean IP, et al. Distal metaphyseal fractures of tibia with minimal involvement of ankle: classification and treatment by locked intramedullary nailing. $\mathrm{J}$ of bone joint surgery British. 1995;77; 781-787.

8. Borelli J Jr, Prickett W, Song E, Becker D, Ricci W (2002). Extraosseous blood supply of tibia and the effect of different plating techniques: a human cadaveric study. Journal of Orthopaedic Trauma 16 (10): 691-695.

9. Aso Mohammed, Ramaswamy Sarawan, Jason Zammit, Richard King. Intramedullary nailing in distal third tibial fractures: distal locking screws and fracture nonunion.

10. Guo JJ, Tang N, Yang HL, Tang TS. A prospective, randomised trial comparing closed intramedullary nailing with percutaneous plating in the treatment of distal metaphyseal fractures of the tibia. J Bone Joint Surg Br. 2010 Jul;92(7): 984e8.

11. Li Yong, Liu Lei, Tang Xin, Pei Fuxing, Wang Guanglin, Fang Yue, Zhang Hui, Crook Nicolas. Comparison of low, multidirectional locked nailing and plating in the treatment of distal tibial metadiaphyseal fractures. Int Orthop (SICOT) 2012;36: 1457e62.

12. Puno RM, Vaughan JJ, Stetten ML, et al. Long term effects of angular malunion on the knee and ankle joints. J of Orthop Trauma 1991; 3:247-54. 